\title{
The effect of age-related IGF-1 deficiency and the occurrence of cardiometabolic risk factors in the Polish population
}

\author{
Aleksandra Zebrowska ${ }^{1}$, Jaroslaw Derejczyk ${ }^{2}$, Barbara Klapcinska ${ }^{1}$ \\ ${ }^{1}$ Department of Physiological and Medical Sciences, The Jerzy Kukuczka Academy \\ of Physical Education, Katowice, Poland. \\ ${ }_{2}^{2}$ John Paul II Geriatric Hospital, Katowice, Poland
}

Keywords: cardiovascular risk management, insulin/IGF-1 pathway, longevity

\section{INTRODUCTION}

Literature data provide evidence of significant age-related decline in circulating levels of insulin like growth factor-1 (IGF1) $[1,2]$. The paracrine IGF-1 system confers vasoprotection and cardioprotection effects and contributes to maintenance of the functional integrity of the neuromuscular system [3, 4]. The aim of the present study was to identify the association between the age-related decline in IGF-1 secretion with the occurrence of cardiometabolic risk factors (CMRFs) in a population-based sample of Polish older adults.

METHODS:

Anthropometric and biochemical parameters were assessed in 982 participants (aged 55-104 years), a population-based sample of Polish older adults. The presented data were collected from a nationwide, multicentre PolSenior Study conducted from 2007 to 2011. In all subjects serum contents of glucose, insulin, total and HDL cholesterol (TC and HDL-C), triglycerides (TG), IGF-1, and IGFBP-3 were measured. To determine IGF-1 bioavailability, the IGF-1/IGFBP-3 molar ratio was calculated. In order to evaluate the risk for cardiometabolic disease, lipid ratios (TC/HDL-C, LDL-C/HDL-C, TG/HDL-C), atherogenic index of plasma (AIP) and insulin resistance index (HOMA-IR) were calculated. Variables of lipid profile, HOMA-IR and somatic variables were used to identify individuals with any CMRF. The Statistica package v. 12 (StatSoft Poland, 12.0) was used for data processing and analyses.

RESULTS:

The results of the study have demonstrated differences in selected cardiometabolic risk factors levels in response to age and gender. Serum IGF-1 concentrations significantly decrease with age $(F=5.7 p<0.001)$. Higher serum IGF-1 and lower IGFBP-3 levels were found in male compared to female subjects and significant associations were observed between HOMA-IR and IGF-1 levels $(F=7.0 \mathrm{p}<0.01)$. Individuals with higher IGF-1/IGFBP-3 ratio $(\mathrm{p}<0.05)$ and higher serum IGF-1 contents were characterized by lower HOMA-IR levels. A significantly higher serum IGF-1 and IGF-1 bioavailability were associated with lower level of insulin resistance, a favorable changes in lipid profile, and higher prevalence of normal blood pressure.

CONCLUSIONS: These findings suggest that low IGF-1 levels have been associated with an increased risk of developing cardiovascular disease and diabetes in older adults.

\section{References}

1) Ungvari Z., Csiszar A. The Emerging Role of IGF-1 Deficiency in Cardiovascular Aging: Recent Advances. J Gerontol A Biol Sci Med Sci. 2012;67A(6):599?610.

2) Deak F., Sonntag W. E. Aging, Synaptic Dysfunction, and Insulin-Like Growth Factor (IGF)-1. J Gerontol A Biol Sci Med Sci. 2012;67A(6):611?625.

3) Barbieri M., Gambardella A., Paolisso G., Varricch M. Metabolic Aspects of the Extreme Longevity. Experimental Gerontology 2008:43:74?78.

4) Chen W., Salojin KV., Mi QS., et al. Insulin-like Growth Factor (IGF)-I/IGF-Binding Protein 3 Complex: Therapeutic Efficacy and Mechanism of Protection Against Type 1 Diabetes. Endocrinology. 2004;145: 627?638. 